\title{
RNA Sequencing for Elucidating an Intronic Variant of Uncertain Significance (SDHD c.314+3A>T) in Splicing Site Consensus Sequences
}

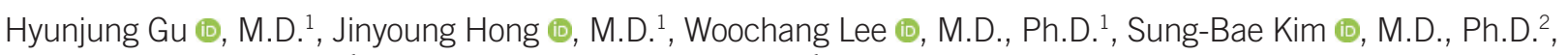
Sail Chun (1), M.D., Ph.D. ${ }^{1}$, and Won-Ki Min (1), M.D., Ph.D. ${ }^{1}$

Departments of ${ }^{1}$ Laboratory Medicine and ${ }^{2}$ Oncology, Asan Medical Center, University of Ulsan College of Medicine, Seoul, Korea

\section{Dear Editor,}

Next-generation sequencing (NGS) is widely used in clinical genetic diagnosis and provides large amounts of genetic information. Efforts have to be made to interpret unrevealed variants simultaneously [1]. Besides the $\pm 1,2$ canonical regions, many variants in introns are interpreted as "variants of uncertain significance" (VUS) because of insufficient evidence of their clinical significance [1]. The best way to confirm variant pathogenicity is RNA sequencing, which can provide evidence of RNA splicing by intronic variants $[1,2]$. Using RNA sequencing, we confirmed that a novel intronic variant (c.314+3A>T) in SDHD induces exon 3 skipping; this case demonstrates the significance of confirming RNA splicing, especially when intronic variants are detected in splice site consensus sequences (ssCSs) [1].

A 41-year-old female patient with a family history of hypertension and paraganglioma was diagnosed as having right carotid paraganglioma in 2001. Multifocal metastases had developed for 21 years. After obtaining informed consent for genetic testing, we performed an NGS hereditary cancer panel testing in 2021 using a MiSeqDx instrument (Illumina, San Diego, CA, USA) and a target enrichment kit (Dxome, Seongnam, Korea). The mean coverage depth was $384.0 \times$. In total, 310 likely benign/ benign variants and a single VUS in SDHD (NM_003002.3: c.314+ $3 \mathrm{~A}>\mathrm{T}$, heterozygous) were detected (Fig. 1).

The VUS has not been detected in the normal population (Genome Aggregation Database; not reported, PM2) and is not reported in ClinVar, the Human Gene Mutation Database, and other databases. Using the splicing computational tool Splicing Site Finder-like (change threshold of $-5 \%$ at donor sites), this variant had a negative score $(-13.6 \%)$, indicating disturbance of normal splicing binding at the donor site (c.314G). The conservation scores in PhastCons (1.0; range, 0-1.0) and GERP (1.0; range, $0-1.0)$ for the variant were very high.

We used RNA sequencing to confirm whether the variant would cause aberrant RNA splicing. Total RNA was extracted from leukocytes of a healthy volunteer and the patient using High Pure RNA Isolation Kit (Roche, Indianapolis, IN, USA) and reversetranscribed to cDNA using the RevertAid First Strand cDNA Synthesis kit (Thermo Fisher Scientific, Waltham, MA, USA). Direct sequencing was performed using target-specific in-house primers (5'-GCTCTGTTGCTTCGAACTCC-3' and 5'-ATGGCATGACAAAGCAGAGG-3').

Exon 3 was skipped in the patient, causing a 145-nucleotide deletion in exon 3 (r.170_314del) (Fig. 2). This caused a frame-
Received: July 23, 2021

Revision received: September 2, 2021

Accepted: November 29, 2021

Corresponding author: Woochang Lee, M.D., Ph.D.

Department of Laboratory Medicine, University of Ulsan College of Medicine and Asan Medical Center, 88 Olympic-ro 43-gil, Songpa-gu, Seoul 05505, Korea

Tel: +82-2-3010-4506, Fax: +82-2-478-0884

E-mail: woochang.lee1@gmail.com

\section{(c) (i) (8)}

\section{(C) Korean Society for Laboratory Medicine}

This is an Open Access article distributed under the terms of the Creative Commons Attribution Non-Commercial License (https://creativecommons.org/licenses/by-nc/4.0) which permits unrestricted non-commercial use, distribution, and reproduction in any medium, provided the original work is properly cited. 

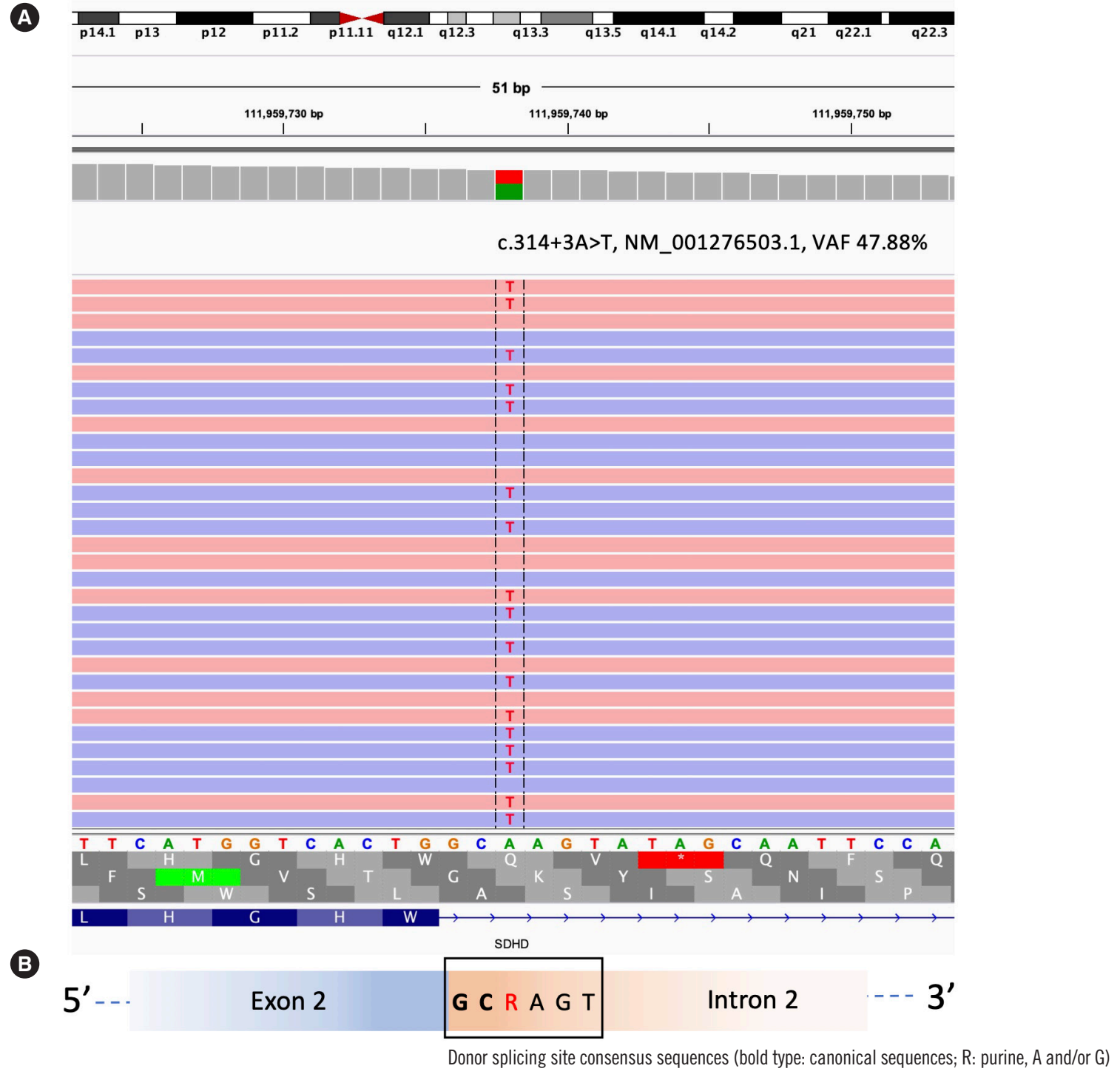

Fig. 1. Representations of genomic alterations in SDHD identified by NGS hereditary cancer panel test (A) Integrative Genomics Viewer (Broad Institute and the Regents of the University of California, https://software.broadinstitute.org/software/igv/home) snapshot of the c.314+3 A > T variant in SDHD (Chr11:111959738, hg19), with a VAF of 47.88\%. (B) Schematic diagram of the donor ssCS in exon 2 in SDHD. Abbreviations: NGS, Next generation sequencing; sSCS, splice site consensus sequence; VAF, variant allele frequency.

shift (p.Ser57TrpfsTer30) that was strongly predicted to have a loss-of-function effect in SDHD. The variant was reclassified as a likely pathogenic variant (PM2, PS3) [3].

The variant c.314+3A $>T$ is detected in highly conserved donor ssCSs recognized by the spliceosomal U1-snRNA complex for splicing (Fig. 1) [4, 5]. Canonical sites at this exon-intron junction have GC sequences (U2 type) instead of the predominant GT sequences at donor splicing sites (U1 type; 5'-GTRAGT-3', where $R$ is a purine $[A$ and/or $G]$ ), and interact with the spliceosomal U1-snRNA, similar to U1-type sequences [6]. This nucleotide alteration from a purine to pyrimidine is presumed to cause exon skipping due to inappropriate donor splice site recognition. Disease-causing spliceogenic variants are reported in canonical sites and adjacent ssCSs of several hereditary cancer genes [1, 2, 7-10]. This suggests the importance of the functional interpretation of intronic variants that cause RNA splicing changes in hereditary cancers. The frequency of intronic variants affecting RNA splicing confirmed by RNA sequencing near acceptor sites, and especially donor sites, is high [1]. RNA sequencing of these sites could reveal valuable information to confirm the pathogenicity of such variants.

A significant number of variants interpreted as VUSs have been 


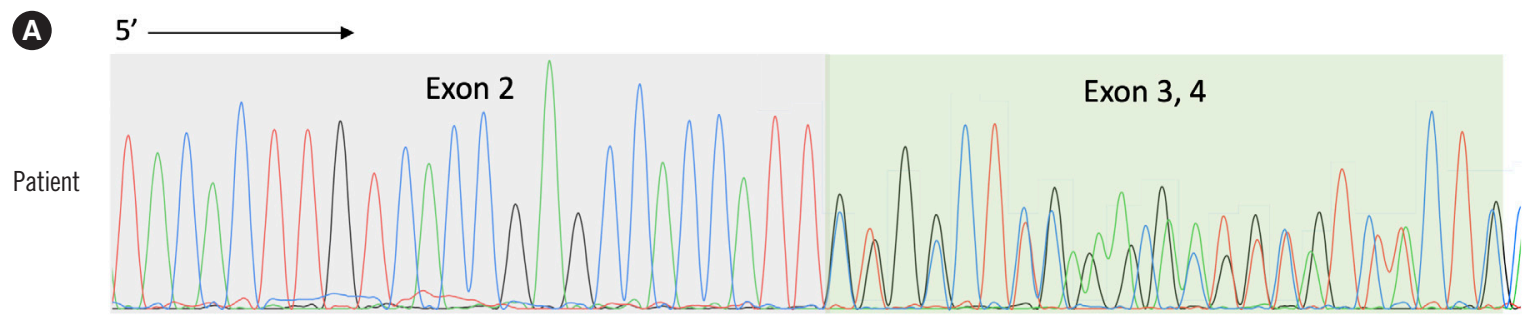

Exon 4 ref.

Exon 2 ref. G G G C C T T G G A A A G T T G T TACT G

TACACTTGTCACCGAGCCACCATT

Exon 3 ref.

CTGGCTCCAAGGCTGCATCTCTC

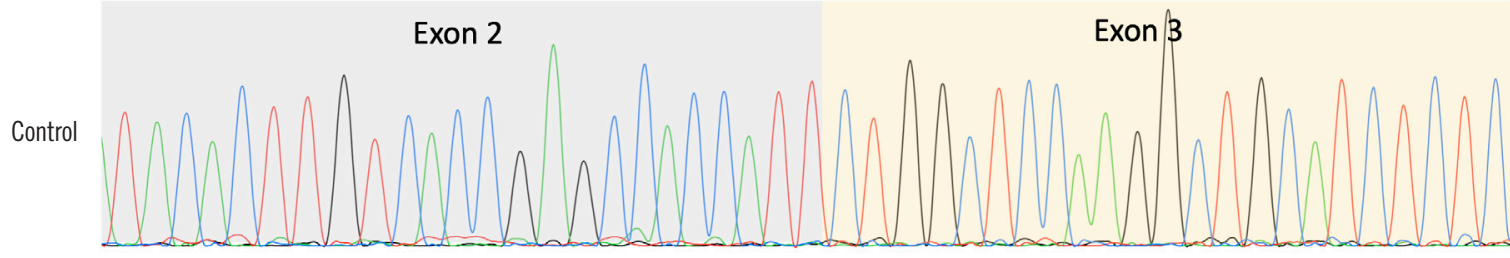

B

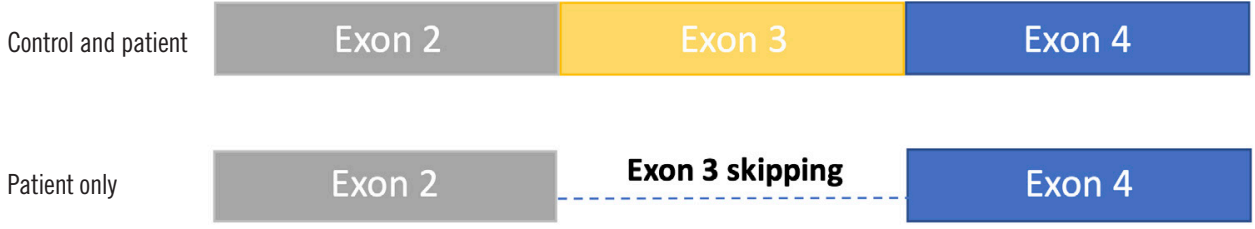

Fig. 2. Schematic diagram of the transcript analysis and sequencing pattern of the control and patient PCR products. (A) RNA sequencing chromatograms (forward sequence) for the patient and control revealing heterozygous exon 3 skipping in the patient. (B) Schematic diagram of transcript analysis of the RNA sequencing results for the patient and control.

reclassified as pathogenic variants via additional RNA sequencing $[1,5,9,10]$. However, many intronic variants tend to be underestimated as VUSs, even if they affect normal RNA splicing [10]. RNA sequencing, when intronic variants are detected in essential ssCSs, could reduce the number of VUSs in intronic regions, enabling more accurate genetic diagnosis and patient management.

\section{ACKNOWLEDGEMENTS}

None.

\section{AUTHOR CONTRIBUTIONS}

Gu H designed the study, analyzed the data, and wrote the draft; Lee $\mathrm{W}$ conceived the study, analyzed the data, and finalized the draft; Kim SB provided clinical information and perspectives on the case; Chun S and Min WK discussed the data and reviewed the manuscript; Hong J discussed the data. All authors read and approved the final manuscript.

\section{CONFLICTS OF INTEREST}

The authors have no conflict of interest to declare.

\section{RESEARCH FUNDING}

None declared.

\section{ORCID}

Hyunjung Gu

https://orcid.org/0000-0001-8673-7326

Jinyoung Hong

https://orcid.org/0000-0001-8855-7900

Woochang Lee

https://orcid.org/0000-0003-3956-6397

Sung-Bae Kim

https://orcid.org/0000-0001-5588-8332

Sail Chun

https://orcid.org/0000-0002-5792-973X

Won-Ki Min

https://orcid.org/0000-0002-5158-2130

\section{REFERENCES}

1. Wai HA, Lord J, Lyon M, Gunning A, Kelly H, Cibin P, et al. Blood RNA 


\section{$\mathrm{Gu} \mathrm{H}$, et al.}

analysis can increase clinical diagnostic rate and resolve variants of uncertain significance. Genet Med 2020;22:1005-14.

2. López-Bigas N, Audit B, Ouzounis C, Parra G, Guigó R. Are splicing mutations the most frequent cause of hereditary disease? FEBS Lett 2005;579:1900-3.

3. Richards S, Aziz N, Bale S, Bick D, Das S, Gastier-Foster J, et al. Standards and guidelines for the interpretation of sequence variants: a joint consensus recommendation of the American College of Medical Genetics and Genomics and the Association for Molecular Pathology. Genet Med 2015;17:405-24.

4. Lewandowska MA. The missing puzzle piece: splicing mutations. Int J Clin Exp Pathol 2013;6:2675-82.

5. Moles-Fernández A, Duran-Lozano L, Montalban G, Bonache S, LópezPerolio I, Menéndez M, et al. Computational tools for splicing defect prediction in breast/ovarian cancer genes: how efficient are they at predicting RNA alterations? Front Genet 2018;9:366.
6. Thanaraj TA and Clark F. Human GC-AG alternative intron isoforms with weak donor sites show enhanced consensus at acceptor exon positions. Nucleic Acids Res 2001;29:2581-93.

7. Bonnal SC, López-Oreja I, Valcárcel J. Roles and mechanisms of alternative splicing in cancer - implications for care. Nat Rev Clin Oncol 2020;17:457-74.

8. Casadei S, Gulsuner S, Shirts BH, Mandell JB, Kortbawi HM, Norquist $\mathrm{BS}$, et al. Characterization of splice-altering mutations in inherited predisposition to cancer. Proc Natl Acad Sci USA 2019;116:26798-807.

9. Lord J, Gallone G, Short PJ, McRae JF, Ironfield H, Wynn EH, et al. Pathogenicity and selective constraint on variation near splice sites. Genome Res 2019;29:159-70.

10. Truty R, Ouyang K, Rojahn S, Garcia S, Colavin A, Hamlington B, et al. Spectrum of splicing variants in disease genes and the ability of RNA analysis to reduce uncertainty in clinical interpretation. Am J Hum Genet 2021;108:696-708. 